\title{
A quest for improving the quality and utility of the individual registry of patients starting renal replacement therapy in Portugal
}

\author{
Edgar A F de Almeida ${ }^{1}$, Anabela Rodrigues ${ }^{2}$ \\ ${ }^{1}$ Nephrology department, Hospital Beatriz Ângelo, Loures \\ ${ }^{2}$ Nephrology department; Hospital Santo António - Centro Hospitalar e Universitário do Porto, Porto
}

Portugal has one of the highest rates of prevalence and incidence of dialysis in Europe. Several reasons have been hypothesized to explain this scenario, ranging from demography, social and economic factors, and ethical issues ${ }^{1}$. Data supporting this picture came from the Annual Report of the Portuguese Society of Nephrology (PSN), covering almost $100 \%$ of information of patients starting and under renal replacement therapy (RRT) since 1997. The report's comprehensive nature and consistency and regularity is one of its major strength and a great achievement of our society.

However, the aggregated nature of the data makes it impossible to ascertain individual outcomes and make appropriate comparisons of patients starting dialysis after a renal transplantation, giving the opportunity to elucidate factors affecting mortality and morbidity of this particular set of patients which recently came to the attention of Portuguese nephrologists ${ }^{2}$. The same is applicable to patients starting peritoneal dialysis and changes between modalities (and reasons for them) or morbidity of patients on dialysis as evaluated by the rate (or time to) hospitalizations.

Since 2009 an individual registry has been set up for patients starting dialysis in Portugal as a part of the reimbursement plan that changed to a bundle payment of dialysis. All patients starting dialysis in Portugal had to be registered through a Nephrology Center in a public hospital which garanteed $100 \%$ registration. In a recent publication $^{3}$, the validity of the individual registry was confirmed by comparing it to the annual report of the PSN. Actually, it was a reciprocal validation of both registries. For the first time it was possible to perform survival analysis of patients starting dialysis in Portugal and to confirm the common assumption that patients starting dialysis in Portugal had similar survival at the $90^{\text {th }}$ day and even slightly better at 1 and at 2 years after the beginning of RRT when compared to the published data of other European countries. However, the absence of key comorbidities hindered adjustments in survival analysis and only crude survival was performed.

Indeed, we are lacking an individual registry of patients with chronic kidney disease for clinical and non-clinical governance.

Only production control and billing of services in hemodialysis have been accomplished to date. Better information management is desirable to allow quality improvement of our health services in nephrology, such as for example: 1 . Survival studies based on therapy modalities and transitions; 2 . Cost-utility studies comparing center dialysis vs home dialysis; 3 . Cost-utility studies comparing hemodialysis in subgroups of elderly patients versus non-dialysis conservative treatment option.

Nephrologists face the redundancy of clinical record supports, the lack of digital systems interaction, the lack of autonomous feeding of the present registry with elected analytical key performance indicators, events of death and its cause, and hospitalization and its cause.

Clinical return of added value for the nephrologists would guarantee adhesion and credibility of the registry. We suggest that the tool should be an activity report periodically sent to the clinical director of the nephrology department or unit including the following critical information:

1. Patients flux

2. Quality strategic criteria such as, for example

1. Glomerular filtration rate at the start of renal replacement therapy

2. Incidence of pmp patients in the referral area

3. Percentage of home dialysis

4. Percentage of live donor transplant

Others quality indicators would be opportune also, according to the properties and the capability of the digital platform:

5. Case mix of the treated population (comorbidity index)

6. Percentage of non-dialysis conservative treatment

7. Percentage of urgent dialysis induction (not programmed)

8. Percentage of induction of hemodialysis with autologous access

9. Survival to 90 days (gross, adjusted)

10. Rate of hospitalizations

11. Mortality associated with dialysis access infection

12. Patient related outcomes

Therefore, it is consensual that the benefits and utility of an individual registry would overcome present limitations and be a source of improving policies.

At this point, if the choice is between creating a new individual registry or improving the existing registry, the latter option is clearly preferable as it avoids the need for authorization of the National Committee for Data Protection. Moreover, as the registry is mandatory for patients starting dialysis in Portugal, this guarantees the inclusion of all dialysis patients. However, improvement in quality of the existent online individual registry needs focused investment by both the 
ministry of health and the nephrology society. Knowledge diffusion and innovation would also benefit from integration of our national individual registry in the individual ERA-EDTA registry. Steps have begun in this direction. Scientific partnership with independent academic institutions in the field of data science and big data management will hopefully create a better future.

Portuguese nephrology is facing an important challenge to step up the quality of the online registry of patients starting dialysis in Portugal, which is decisive to answer some key questions that remains unanswered as well as to improve the quality of nephrology services in a sustainable and innovative way ${ }^{4}$.

Working together, we can achieve this.

\section{References}

1. Stel VS, Bruck K, Fraser S et al. International differences in chronic kidney disease prevalence: a key public health and epidemiological research issue. Nephrol Dial Transplant 2017;32:ii129-ii135. 2. Leal R. The failing renal graft: a new chapter for CKD patients. Port J Nephrol Hypert 2020;34(2):7375.

3. de Almeida E, Raimundo M, Coelho A, Sá H. Incidence, prevalence and crude survival of patients starting dialysis in Portugal (2010-16): analysis of the National Health System individual registry. Clinical Kidney Journal 2020;1-7.

4. van der Tol A, Stel VS, Jager KJ, et al. A call for harmonization of European kidney care: dialysis reimbursement and distribution of kidney replacement therapies. Nephrol Dial Transplant. 2020; 1;35(6):979-986

\section{Correspondence to:}

Edgar Almeida

Nephrology department, Hospital Beatriz Ângelo, Loures

E-mail: edealmeida@mail.telepac.pt 